\title{
Case Report: Neglected Case With Good Outcome Thoracic Psammomatous Spinal Meningioma
}

\author{
Heru Hermantrie ${ }^{1 *}$, Otman Siregar ${ }^{2}$, \\ Pranajaya Dharma Kadar ${ }^{2}$, Heru Rahmadhany ${ }^{2}$, Benny ${ }^{3}$
}

\author{
${ }^{1}$ Resident of Orthopaedic and Traumatology, Faculty of Medicine, Universitas \\ Sumatera Utara, H. Adam Malik Hospital Medan, Indonesia \\ ${ }^{2}$ Consultant of Orthopaedic and Traumatology, Spine Division, Faculty of Medicine, \\ Universitas Sumatera Utara, H. Adam Malik Hospital Medan, Indonesia \\ ${ }^{3}$ Staff of Orthopaedic and Traumatology, Spine Division, Faculty of Medicine, \\ Universitas Sumatera Utara, H. Adam Malik Hospital Medan, Indonesia
}

\begin{abstract}
Introduction: Spinal Meningiomas are most common in adult spinal tumors, account for $25-46 \%$ of tumors of spine with the highest incidence seen in thoracic region of middle-aged-woman.. Advance in radiological and surgical assistive devices have resulted earlier diagnosis. Surgical resection is the standard treatment and now associated with a good postoperative outcome. Case Report:A 47-year-old woman with 2 years history of numbness progressed below the umbilicus. Local pain on her back, walks in creeping way, urinary and fecal incontinence. MRI showing hypointense lesion on level T7, underwent surgical resection and histopathological examination revealed psammomatous meningioma (WHO Grade I). Patient discharged with improvement gait but some residual numbness and weakness on her right foot. Discussion: Meningiomas are primary tumors of spine, account for $25-46 \%$ of tumors of spine with the highest incidence seen in thoracic region of middle-aged-woman. Lead to symptoms only when they reach a considerable size to compress the spinal cord. Surgical resection is standard treatment and is now associated with a good postoperative outcome. Conclusion: Spinal Meningiomas with the highest incidence seen in thoracic region of middle-aged-woman. Present with pain, sensory loss, weakness and spinchter disturbance. MRI is recommended for the diagnosis. Surgical resection is standard treatment and associated with a good postoperative outcome.
\end{abstract}

Key words: Spinal meningioma, Intradural, Extramedullary Meningioma.

Heru Hermantrie et al / International Journal of PharmTech Research, 2019,12(2): 134-138.

DOI: http://dx.doi.org/10.20902/IJPTR.2019.120206 


\section{Introduction ${ }^{1,2}$ :}

Spinal meningiomas account for $25 \%-46 \%$ of all intraspinal tumor. Spinal meningiomas are slow growing tumors and therefore, they lead to symptoms only when they reach a considerable size to compress the spinal cord, causing local pain. Intraspinal meningiomas are most commonly located in thoracic region, followed by cervical and lumbar areas. More common in elderly patients Tumor location and size are the major factors to consider in complete tumor resection.

\section{Case Report:}

A 47-year-old woman complained of 2 years history of numbness progressed below the umbilicus. The patient also noted progressive weakness in his lower extremities. The patient walks in creeping way, urinary and fecal incontinence. The patient's past lower motor examination showed $(0 / 5)$ both legs and neurological examination revealed paraparesis below the T7 level. Lower extremity reflexes knee and ankle jerk reflex exaggerated, bilateral ankle clonus present and tone in lower limb increased. Upper limb examination was unremarkable.

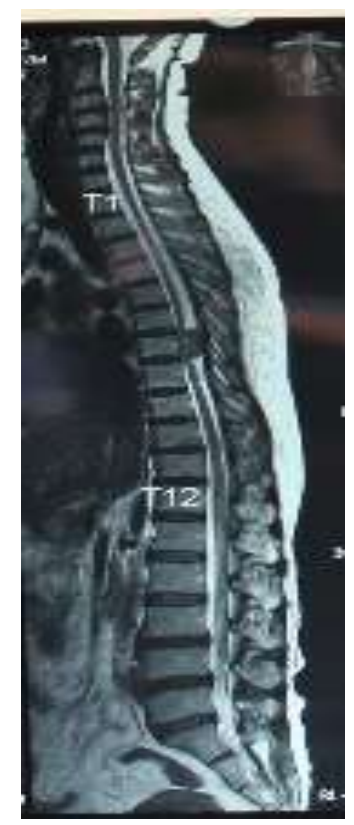

Fig. 1. T2 weighted sagital view showed hypointense lesion on level T7 compressing of the cord

Thoracic magnetic resonance imaging showed a $2 \mathrm{~cm}$ hypointense lesion on T1-weighted and T2-weighted on level T7 and causing compression of the cord.

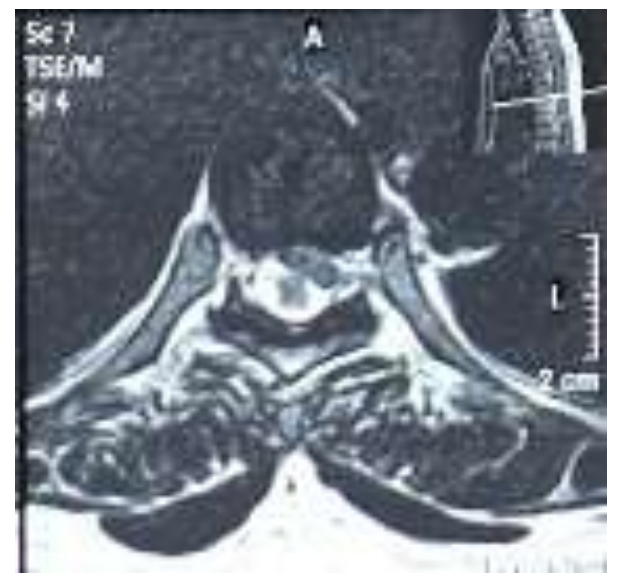

Fig. 2. T2 weighted axial view showed lesion on intradural 
Based on clinicoradiological correlation, patient underwent a T7 laminectomy using operative microscope through posterior midline incision. After laminectomy, the duramater was exposed and incised at midlne, exposing an extramedullary mass compressing the spinal cord.

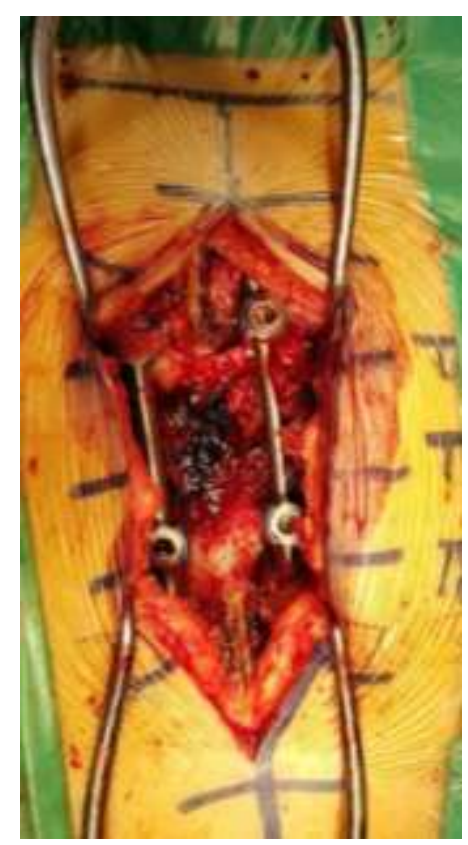

Fig. 3. During Operation and stabilizing using rods

Using the microscissors and microdissector, the tumor were removed from spinal cord. After removal the tumor, the margin of the resected inner layer was coagulated using bipolar cauterization. Rods was applied to maintain the stability.

The excised tumor mass was subjected to histopathological examination. The tumor composed predominantly of psammoma bodies, eosinophilic cytoplasm, and proliferation cell with round to oval core. Final impression was psammomatous meningioma WHO grade I.

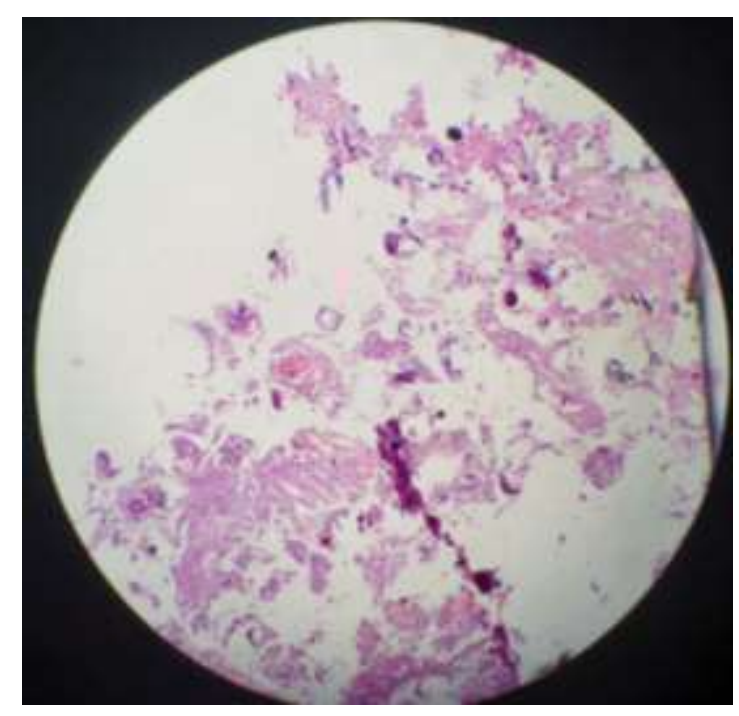

Fig. 4. Histological finding composed predominantly of psammoma bodies 
Patient was in follow up and after 4 months, clinically have improvement from grade 0 muscle power to 5 but still using walker frame and exhibit mild residual numbness and weakness on her right foot. Bladder dysfunction was cured.

\section{Discussion $^{2-7}$ :}

Meningiomas are primary tumors of spine, arisen from the arachnoid cap cells of the meningens. Spinal meningiomas account for $25 \%-46 \%$ of all intraspinal tumors.

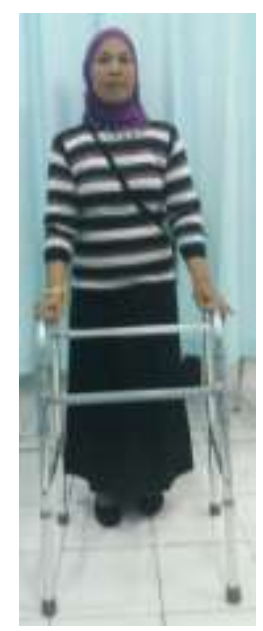

Fig. 5. Clinical appearance after 4 months postoperative

They are typically classified as intradural extramedullary spinal tumors. The incidence of intradural spinal tumors is reported to be between 1 and 2 per 100.000 population. Spine meningiomas have high predilection for women, most often affect middle-aged women. The female / male ratio is between 3-4.3: 1 (mean age range 49-62yo). In the study of Roux and Hanieh study, sex and gender has no influence on the prognosis. Meningiomas of the spine occur in the thoracic spine in approximately $80 \%$, followed in frequency by the cervical and lumbar regions. In the Schaller study, tumor location below $\mathrm{C} 4$ seemed to be correlated with a good outcome. Spinal meningiomas are slow growing tumors and therefore, lead to symptoms only when they reach a considerable size to compress the spinal cord, causing local pain. Patient typically present with pain, sensory loss, weakness and spinchter disturbance.

Concerning neuroimaging, MRI is recommended for the diagnosis of spinal meningiomas. MRI of spinal meningiomas on T1-weighted images exhibit signal intensity similar to that of spinal cord and does not show significant increased signal intensity in T2-weighted images.

Histopathology of spinal meningiomas usually exhibits meningothelial, fibroblastic, transitional and psammomatous pattern. Meningioma is surgically-treated disease. In Roux study, the majority of the neoplasm were meningothelial $(\mathrm{N}=24)$ or psammomatous $(\mathrm{N}=11)$ with ni mortality noted. Final functional were very good and 2 recurrences was noted and underwent complementary radiotherapy. In Ucler study, all patients underwent traditional posterior approach noted none of the cases demonstrated postoperative neurological deterioration and postoperative spinchter dysfunction.

In Gotfried et $\mathrm{al}^{3}$ study from the patient who suffered 4-month history of numbness and progressive weakness in his lower extremity, underwent gross total resection of tumor. Day-4 postoperative, the patient ambulated with a cane and regained full urinary and bowel continence after 2 months follow up.

Hirabayashi et $\mathrm{al}^{4}$ reported an 82-year old woman who complained 1-year history of leg pain and numbness and developed urinary incontinence, underwent laminectomy and tumor resection through posterior midline incision. Day-3 postoperative have markedly decrease of pain and numbness and ambulate independently 4 days after surgery without tumor recurrence showed on MRI after 5 years. 
Surgical resection is standard treatment and is now associated with a good postoperative outcome. Alternatively, a wide dural excision and simple duraplasty can be performed. Surgical excision was graded according to Simpson's classification, tumors known to have undergone Simpson's grade 1 or 2 surgical removal were designated as total resection and grades 3-5 as subtotal resection. In such cases, total removal must not be an absolute surgical goal. In Roux study, three of four patients who had a partial removal did not present any recurrence ${ }^{7}$.

The recurrence rate associated with subtotal resection is significantly higher than that associated with complete resection. In Roux study, from 2 patients underwent subtotal tumor removal presented with a recurrence 2 years after primary surgery continued by radiation and present with normal neurologic findings and slight disability in walking ${ }^{7}$. The major clinical factor in recurrence is the extent of resection which is invariably influenced by tumour site, extent of invasion and attachment to vital intracranial / intraspinal structure. Benign meningiomas have recurrence rates approximately $7-25 \%$, atypical meningiomas show recurrence rate of $29-52 \%$ and anaplastic meningiomas recur in 50-94\% of cases. Meningiomas with malignant histological features are associated with shorter survival times, approximately 2-5 years depending largely on the extent of resection.

\section{Conclusion:}

Intradural Psammomatous meningiomas are very rare lesions. Patient typically present with pain, sensory loss, weakness and spinchter disturbance. MRI is recommended for the diagnosis of spinal meningiomas. Surgical resection is standard treatment and is now associated with a good postoperative outcome. Alternatively, a wide dural excision and simple duraplasty can be performed. Its goal should be radical resection of the lesion to avoid recurrence.

\section{References:}

1. Rothman-Simeone; The Spine; Sixth edition, volume one,page 1612-1623 Elsevier Saunders 2011.

2. Prakash Akhil, Mishra Shashank, Tyagi Ruchi, Kansal Sandeep. Thoracic psammomatous spinal meningioma with osseous mepatplasia: A very rare case report. Asianjs published by wolter Kluwer. Volume 12. April - June 2017

3. Gottfried N Oren, Gulf Wayne, Kan Peter. Spinal Meningiomas: Surgical Management and outcome. Neurosurgical Focus. 14. Volume 14. June 2003

4. Hirabayashi Hiroki, Takahashi Jun. Surgcal resection without dural reconstruction of lumbar meningioma in elderly woman. European Spine Journal.2009

5. Davies Simon, Gregson Barbara. Mitchell Patrick. Spinal Meningioma: Relationship between degree of cord compression and outcome. British Journal of Neurosurgery. 2016

6. Schaller B. Spinal Meningioma: Relationship between histological subtypes and surgical outcome? Journal of Neuro-Oncology, 2005doi:10.1007/s11060-005-1469-4.

7. Roux FX, Nataf F, Pinaudeau M, Borne G, Devaux B, Meder JF. Intraspinal meningiomas: review of 54 cases with discussion of poor prognosis factors and modern therapeutic management. Surgical Neurology. 1996doi:10.1016/S0090-3019(96)00199-1. 